\title{
Nitroglycerin Transdermal Patch
}

National Cancer Institute

\section{Source}

National Cancer Institute. Nitroglycerin Transdermal Patch. NCI Thesaurus. Code C103272.

A sustained release transdermal patch containing the organic nitrate nitroglycerin, with vasodilator and potential immunomodulating activities. Upon application to the skin, nitroglycerin is continuously released from the patch and absorbed. In turn, nitroglycerin is converted into nitric oxide (NO), which activates guanylyl cyclase, increasing cyclic guanosine monophosphate concentration thus resulting in smooth muscle relaxation. In addition, activation of NO-mediated signaling pathways may inhibit hypoxia-induced tumor cell invasiveness, chemoresistance, evasion of immune cell recognition and cancer cell progression. Particularly, reactivation of NO-mediated signaling appears to inhibit the increased tumor cell shedding of the major histocompatibility complex class I chainrelated (MIC) molecules MICA and MICB as is seen in hypoxic tumor environments; MIC molecules play key roles in tumor cell immune surveillance through their interaction with the C-type lectin-like NKG2D receptor on natural killer, lymphokine-activated killer and effector T cells. 